\title{
APLICAÇÃO DE FÁCIES ORGÂNICA NA ANÁLISE PALEOCEANOGRÁFICA DO TALUDE CONTINENTAL SUPERIOR RECENTE DA BACIA DE CAMPOS, RJ, BRASIL
}

\author{
TAÍSSA RÊGO MENEZES \\ GEO/CENPES/PETROBRAS, Ilha do Fundão, 21949-900, RJ, Brasil.taissar.fundacao_padre@petrobras.com.br \\ JOÃO GRACIANO MENDONÇA-FILHO \\ DEGEO/IGEO/UFRJ, Ilha do Fundão, 21949-900, RJ, Brasil. graciano@geologia.ufrj.br
}

RESUMO - Uma seção sedimentar quaternária do talude continental superior da Bacia de Campos amostrada do testemunho de sondagem BU-91-GL-05, foi analisada com o objetivo de determinar as variações dos parâmetros palinofaciológicos e organogeoquímicos. O testemunho de sondagem BU-91-GL-05 foi coletado em uma lâmina d'água de 630 m e possui 149,5 m de comprimento, compreendendo uma sucessão sedimentar com o predomínio de lamas, fácies arenosas e depósitos de movimento de massa. Um estudo qualitativo e quantitativo da matéria orgânica foi realizado em 38 amostras coletadas deste testemunho. As amostras foram processadas seguindo os padrões de preparação para palinofácies. Os resultados percentuais obtidos foram submetidos a análises de agrupamento (modo- $Q$ para observação de similaridades entre as amostras, e modo- $R$ para verificar similaridades entre os componentes orgânicos). Além disso, foram realizadas análises de Carbono Orgânico Total (COT) e Pirólise Rock-Eval. As amostras em geral apresentaram o predomínio do grupo dos fitoclastos, seguido do grupo de palinomorfos e matéria orgânica amorfa. A análise de agrupamento (modo- $R$ ), reuniu os componentes orgânicos particulados em 4 sub-grupos. Estes dados refletem as características hidrodinâmicas e tendências proximais-distais das partículas orgânicas. A análise de agrupamento modo- $Q$ determinou 6 intervalos, sendo estes correlacionáveis com períodos interglaciais e glaciais pré-definidos para o Pleistoceno. Esses intervalos apresentam suprimento variado de matéria orgânica continental e marinha. A faciologia orgânica caracterizou variações paleoambientais desde fácies proximal de plataforma óxica até o intermediário-distal de fácies de talude com regime disóxico. As variações dos parâmetros palinofaciológicos e organogeoquímicos estão relacionadas às oscilações do nível relativo do mar, reflexo de flutuações climáticas, ocorridas nos últimos 145.000/150.000 anos AP, que afetaram os fatores gerais que produziram, acumularam e preservaram os grupos e subgrupos de componentes da matéria orgânica.

Palavras-chave: palinofácies, fácies orgânica, paleoceanografia, talude continental superior recente, bacia de Campos.

ABSTRACT - APPLICATION OF ORGANIC FACIES IN THE PALEOCEANOGRAPHIC ANALYSIS FROM UPPER CONTINENTAL SLOPE OF CAMPOS BASIN, RJ, BRAZIL. The Quaternary section from BU-91GL-05 well from upper continental slope of Campos Basin were studied aiming its palynofacies and organic facies characterization. The sedimentary sucession recovered from BU-91-GL-05 well is located on upper continental slope and comprises 149,5 m length at water of depth $630 \mathrm{~m}$. The studied section comprises mainly mud facies, sand facies and also mass movement deposits. The geochemical analyses included Total Organic Carbon (TOC) and Rock-Eval Pyrolysis determinations. Thirty-eight samples were analyzed by this method and final data were submitted to cluster analyses ( $r$ and $q$-ratio). The cluster analysis was based on abundance of organic particles in order to define groupings (similarity) and to recognize the relationship between palynofacies analysis. In general, the organic matter assemblage presents a predominance of phytoclasts (woody organic matter), moderate percentage of palynomorphs, and low to moderate percentage of amorphous organic matter. $R$-mode cluster analysis revealed four sub-groups. These results reflect proximal-distal trends and hydrodinamic equivalence effect. $Q$ - mode analyses revealed six intervals to the sedimentary section from BU-91-GL-05 well. These groupings can be correlated with glacial/interglacial periods defined for the Pleistocene age. These intervals were represented by high/low supply of terrestrial organic matter and marine organic matter. The palynofacies and organic facies parameters suggest paleoenvironmental variations in the studied sections (from a proximal oxidizing to distal dysoxic environment). These variations may be correlated to climatic events and sea level changes occurred at 145.000/150.000 years BP, that influenced the production, accumulation and preservation of organic matter from upper continental slope of the Campos Basin.

Key words: palynofacies, organic facies, paleoceanography, upper continental slope, Campos basin. 


\section{INTRODUÇÃO}

O talude continental brasileiro recente foi intensamente estudado no que diz respeito à sedimentologia, morfologia e hidrologia (Viana et al.,1998; Viana \& Faugerés, 1998; Caddah et al. 1998). Em relação a estudos de componentes orgânicos particulados, Henz et. al, 1996 realizaram estudos de petrografia orgânica em amostras com o objetivo de investigar os parâmetros capazes de influenciar as propriedades geotécnicas de solos marinhos, onde observaram o predomínio de material orgânico de origem de vegetais terrestres superiores.

A determinação dos parâmetros palinofaciológicos e organogeoquímicos e suas variações no talude recente são uma valiosa ferramenta na reconstrução paleoambiental do Talude Continental Brasileiro Recente na Bacia de Campos, com aplicação imediata na construção de modelos preditivos a serem aplicados em outros períodos geológicos.
Viana \& Fabèrges (1998) descrevem que os sedimentos depositados na plataforma externa e talude continental superior na Bacia de Campos têm sido retrabalhados ao longo da plataforma e dirigidos ao talude por forças oceanográficas de borda de plataforma e trapeadas pela morfologia local. Os autores consideram que a fisiografia local, fácies sedimentares, características hidrodinâmicas e o impacto de flutuações relativas do nível do mar influenciam corpos de areia que se estendem além de 700 $\mathrm{m}$ de lâmina d'água. Desta forma, os fatores geológicos envolvem a fisiografia da área, o aporte sedimentar e a granulometria das partículas.

Os componentes orgânicos são considerados hidrodinâmicamente correlacionados as partículas sedimentares. Por esse motivo, o estudo da assembléia da matéria orgânica fez-se necessário no presente estudo, pois o transporte de sedimentos e da matéria orgânica da plataforma continental até o oceano envolve fatores geológicos e oceanográficos, nos quais oscilações relativas do nível do mar estão incluídas.

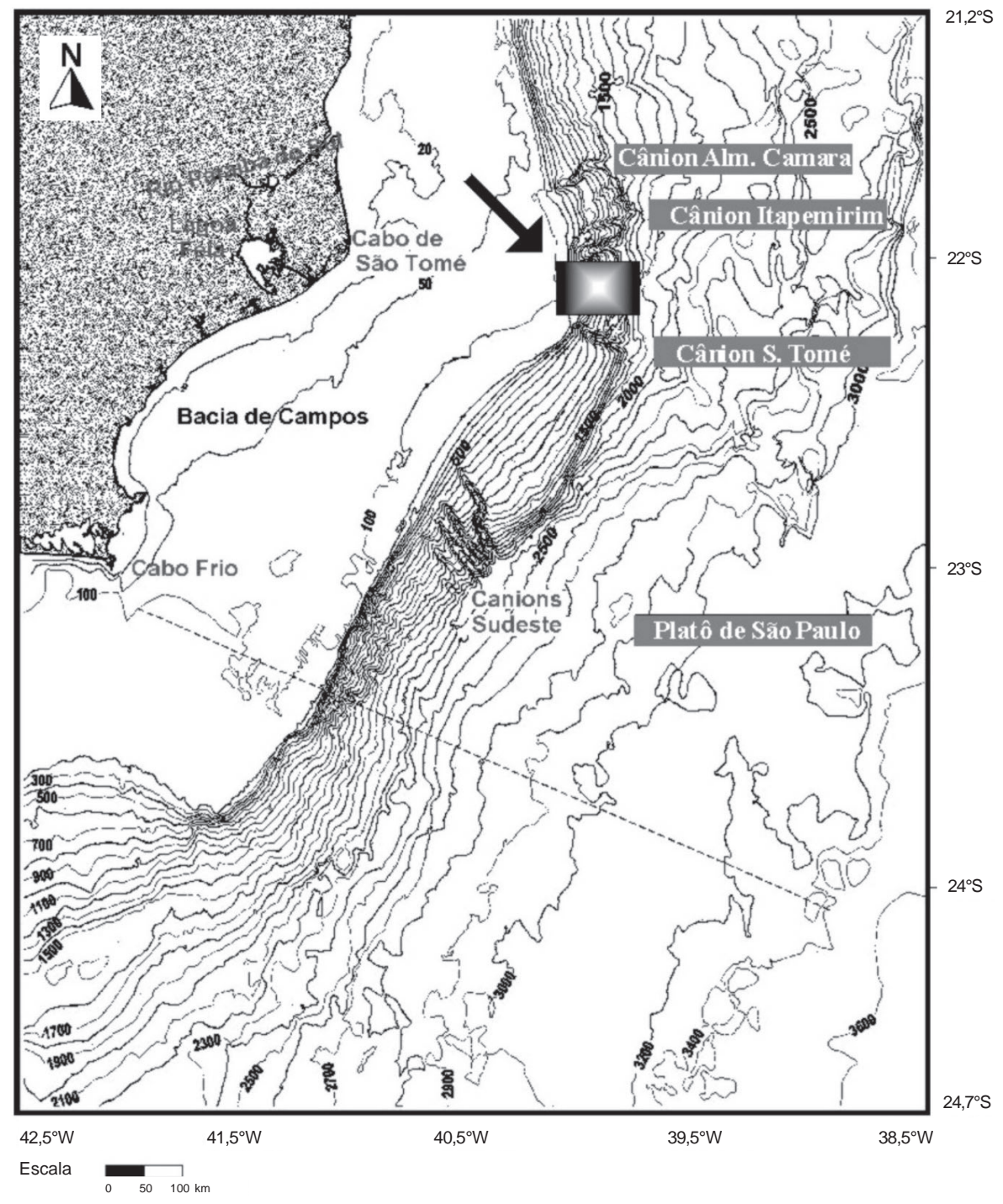

Figura 1. Mapa de localização do testemunho de sondagem BU-91-GL-05 (modificado de Viana et al. 1998). Figure 1. Location map of the BU-91-GL-05 well (modified from Viana et al., 1998). 
Este estudo compreendeu a aplicação de palinofácies e de fácies orgânica, utilizando técnicas de microscopia em luz branca transmitida e luz azul incidente (fluorescência) e métodos geoquímicos (Carbono Orgânico Total e pirólise Rock-Eval) para a identificação dos diferentes grupos e subgrupos de componentes da matéria orgânica (componentes orgânicos particulados).

As informações adquiridas por estas técnicas foram avaliadas no amplo contexto dos fatores gerais que controlam a sedimentação, distribuição e preservação de matéria orgânica. Por sua vez, a análise palinofaciológica dos componentes da matéria orgânica produz um nível considerável de resoluções paleoambientais e paleoceanográficas, que são baseadas nos procedimentos de contagem adequados dos componentes orgânicos particulados.

\section{MATERIAL E MÉTODOS}

A área de estudo localiza-se entre os paralelos $22^{\circ}$ e $22^{\circ} 30^{\prime}$ S na costa do Estado do Rio de Janeiro. Esta região é cortada pelo Cânion São Tomé e limitada ao norte pelo Cânion Itapemirim (figura 1) e compreende a uma seção quaternária do talude continental superior.

Esta região caracteriza-se pela forte influência de correntes como a Corrente do Brasil (Western Boundary Currents STW), a sua contra-corrente Contra Corrente do Brasil (South Atlantic Central Waters - SACW) e está sob o padrão de circulação profunda e bem ventilada (oxigenada) (Viana et al.,1998; Henz et al., 1996).

Foram analisadas 38 amostras coletadas do testemunho de sondagem BU91-GL-05 que foram cedidas pela PETROBRAS para o projeto CNPQ-CTPETRO/UFRJ “Aplicação da Faciologia Orgânica na Modelagem Paleoceanográfica e Microbiofaciológica do Talude Continental Brasileiro Recente da Bacia de Campos - RJ". O testemunho de sondagem estudado apresenta 149,5m de comprimento e foi coletado sob lâmina d'água de 630 metros.

No presente trabalho adotou-se de maneira simplificada o zoneamento bioestratigráfico e paleoclimático baseado em foraminíferos planctônicos feito por Vicalvi (1999) para testemunho de sondagem analisado (BU-91-GL-05).

O zoneamento feito por Vicalvi (1999) permitiu uma rápida e confiável divisão do quaternário do talude da Bacia de Campos, onde foram identificadas 4 zonas: Z, Y, X, W e delineados claramente dois episódios glaciais (zonas Y e W) e dois interglaciais (zonas $\mathrm{Z}$ e X). Desta forma, a sucessão sedimentar analisada representa os sedimentos depositados provavelmente, nos últimos 145.000/150.000 anos A.P(Vicalvi, 1999). Segundo Caddah et al. (1998) a seção sedimentar é composta por aproximadamente $80 \%$ de fácies lamosas moderadamente a intensamente bioturbadas, fácies arenosas e depósitos de movimento de massa.

Segundo Tyson (1995), a análise de palinofácies é o estudo palinológico de ambientes deposicionais e do potencial de rochas geradoras de hidrocarbonetos baseado na assembléia total de componentes da matéria orgânica particulada. Palinofácies pode ser definida como "um corpo de sedimen- to contendo uma assembléia distinta de matéria orgânica palinológica que reflete um grupo específico de condições ambientais, podendo ser associada com um potencial de geração de hidrocarbonetos característico".

Fácies orgânicas têm sido descritas em rochas de diversas idades em várias áreas. O critério químico é primariamente usado para caracterizar fácies orgânicas que refletem processos químicos, físicos e biológicos (Jones, 1987). Tuweni \& Tyson (1994) consideram que a combinação de COT e pirólise RockEval com análises de palinofácies se constitui em um modo excelente de caracterização de fácies orgânica. Desta forma, neste trabalho foi adotado a aplicação de fácies orgânica segundo Jones (1987) e suas relações com a palinofácies de Tyson (1995).

A preparação deste material para a análise de palinofácies utilizou os procedimentos palinológicos padrões não oxidativos descritos por Tyson (1995) e Mendonça Filho (1999) e o Sistema de Neutralização de Resíduos Ácidos de Assis (2000) para isolamento da matéria orgânica. As amostras foram tratadas com ácido clorídrico e fluorídrico para eliminação da fração mineral, e a separação da matéria orgânica do resíduo mineral e sua concentração foi realizada com a utilização de cloreto de zinco. A matéria orgânica isolada foi então montada em lâminas de vidro para as análises em luz branca transmitida e fluorescência. As análises de COT foram realizadas no determinador LECOSC-44 e as análises de pirólise foram obtidas pelo equipamento Rock-Eval 6.

A técnica de palinofácies envolveu o exame qualitativo e quantitativo tanto da matéria orgânica total quanto na distribuição de suas diversas classes nos sedimentos estudados e paralelamente sua correlação com as análises organogeoquímicas. As partículas orgânicas foram designadas através do sistema de classificação (baseado em Tyson, 1995; Mendonça Filho, 1999; Mendonça Filho et al., 2002), sendo o material orgânico dividido em três grupos principais: Matéria Orgânica Amorfa (MOA), Fitoclastos e Palinomorfos (TABELA 1).

Dois sistemas de contagem foram realizados, o primeiro contabilizou o total de matéria orgânica (500 partículas), incluindo os três grupos da matéria orgânica e seus subgrupos, e o segundo contabilizou o total da fração palinomorfo (300 partículas). No primeiro sistema, fez-se à cobertura da lâmina utilizando um retículo cruzado graduado em oculares de $10 \mathrm{X}$ e objetiva de $20 \mathrm{X}$ de aumento. Foram registradas somente aquelas partículas que passaram diretamente sobre o retículo. No segundo sistema, contagem da fração palinomorfo, foram registradas todas as partículas que caíram no campo visual.

Após a contagem das partículas orgânicas foi realizado tratamento estatístico com os dados absolutos obtidos. Estes dados absolutos foram recalculados para valores percentuais relativos aos grupos e subgrupos da matéria orgânica e submetidos a análises de agrupamento modo- $Q$ e o modo- $R$. Além disso, foi empregado o coeficiente de correlação linear $r$ de Pearson ( $r$-Pearson) para quantificar a dependência linear entre os elementos e, assim determinar a 
Tabela 1. Classificação geral dos principais componentes da matéria orgânica (baseado em Tyson 1995; Mendonça Filho, 1999; Mendonça Filho et. al., 2002).

Table 1. Classification of organic matter components (based on Tyson 1995; Mendonça Filho, 1999; Mendonça Filho et. al., 2002).

\begin{tabular}{|c|c|c|c|c|}
\hline \multicolumn{2}{|c|}{ GRUPOS \& SUBGRUPOS } & ORIGEM & \multicolumn{2}{|c|}{ DESCRIÇÃo } \\
\hline \multirow[b]{2}{*}{$\begin{array}{l}\text { Matéria } \\
\text { Orgânica } \\
\text { Amorfa }\end{array}$} & "MOA" & $\begin{array}{l}\text { Material orgânico derivado de } \\
\text { ataque microbiológico }\end{array}$ & \multicolumn{2}{|c|}{$\begin{array}{l}\text { Material não estruturado e com formato variado; cor: amarelo- } \\
\text { laranja-vermelho; laranja-marrom; cinza, algumas vezes inclusõos } \\
\text { como palinomorfos, fitoclastos, pirita, etc; fluorescente a não- } \\
\text { fluorescente. }\end{array}$} \\
\hline & Resina & $\begin{array}{c}\text { Derivado de vegetais terrestres } \\
\text { superiores de florestas tropicais } \\
\text { e subtropicais }\end{array}$ & \multicolumn{2}{|c|}{$\begin{array}{l}\text { Partícula não estruturada, hialina, usualmente arredondada, } \\
\text { homogênea, fluorescente a não-fluorescente. }\end{array}$} \\
\hline \multirow{7}{*}{ Fitoclastos } & \multirow[b]{2}{*}{ Opaco } & Equidimensional & \multirow{7}{*}{$\begin{array}{l}\text { Derivado de tecidos } \\
\text { lenhosos de vegetais } \\
\text { superiores ou fungos }\end{array}$} & $\begin{array}{l}\text { Partícula de cor preta de forma } \\
\text { quadrática. Sem bioestruturas internas. }\end{array}$ \\
\hline & & Alongado & & $\begin{array}{c}\text { Partícula de cor preta de forma } \\
\text { alongada. Eixo longo três vezes mais } \\
\text { do que o eixo curto. Sem bioestrutura } \\
\text { interna. }\end{array}$ \\
\hline & \multirow{5}{*}{ Não-Opaco } & Não-bioestruturados & & $\begin{array}{l}\text { Partícula de cor marrom não } \\
\text { bioestruturada }\end{array}$ \\
\hline & & Bioestruturados & & $\begin{array}{l}\text { Partícula de cor marrom bioestruturada } \\
\text { (estriada, listrada, bandada, perfurada) }\end{array}$ \\
\hline & & Cutículas & & $\begin{array}{l}\text { Partículas de cor amarelo-pálido a } \\
\text { marrom claro, delgadas, com estrutura } \\
\text { celular, em alguns casos com } \\
\text { estômatos visíveis. } \\
\end{array}$ \\
\hline & & Membranas & & $\begin{array}{c}\text { Partículas de cor amarelo-pálido, } \\
\text { comumente transparentes, sem } \\
\text { estrutura celular. }\end{array}$ \\
\hline & & Hifas de Fungo & & $\begin{array}{l}\text { Filamentos individuais do micélio da } \\
\text { fase vegetativa dos fungos. }\end{array}$ \\
\hline \multirow{7}{*}{ Palinomorfos } & \multirow[b]{2}{*}{ Esporomorfo } & Esporo & $\begin{array}{l}\text { Palinomorfo terrestre } \\
\text { produzido por pteridófitos, } \\
\text { briófitas e fungos. }\end{array}$ & $\begin{array}{l}\text { Palinomorfo de forma triangular ou } \\
\text { circular, apresentando a marca trilete } \\
\text { ("Y") ou monolete (uma cicatriz) } \\
\text { Ornamentação variada. }\end{array}$ \\
\hline & & Grão de Pólen & $\begin{array}{l}\text { Palinomorfo terrestre } \\
\text { produzido por } \\
\text { gimnospermas e } \\
\text { angiospermas } \\
\end{array}$ & $\begin{array}{l}\text { Palinomorfo com morfologia } \\
\text { complexa a simples; usualmente } \\
\text { esférico a subesférico; ornamentaçãp } \\
\text { variada; pode apresentar aberturas. } \\
\end{array}$ \\
\hline & \multirow[b]{2}{*}{$\begin{array}{l}\text { Microplâncton de } \\
\text { água doce }\end{array}$} & Botryococcus & \multirow[b]{2}{*}{ Alga Chlorococcale } & $\begin{array}{l}\text { Colônias globular irregular; tamanho } \\
30 \text { a } 2000 \text { ? m, algumas vezes com } \\
\text { vários lóbulos; laranja-marrom. } \\
\text { Carbonífero-Recente. } \\
\end{array}$ \\
\hline & & Pediastrum & & $\begin{array}{l}\text { Algas verdes coloniais, radialmente } \\
\text { simétricas; tamanho } 30-200 ? \mathrm{~m} \text { em } \\
\text { diâmetro e com um dois chifres no } \\
\text { anel mais externo das células. Células } \\
\text { internas podem ser de forma irregular } \\
\text { com espaços entre elas ou } \\
\text { compactadas. Jurássico?-Recente. }\end{array}$ \\
\hline & \multirow[t]{2}{*}{$\begin{array}{l}\text { Microplâncton } \\
\text { marinho }\end{array}$} & Dinoflagelados & $\begin{array}{l}\text { Restos de cistos produzidos } \\
\text { durante a parte sexual do } \\
\text { ciclo de vida da Classe } \\
\text { Dinophycea }\end{array}$ & $\begin{array}{l}\text { Principal característica é a } \\
\text { paratabulação que divide a teca do } \\
\text { cisto em placas retangulares ou } \\
\text { poligonal separadas por suturas. Três } \\
\text { morfologias principais: proximados, } \\
\text { cavados e corados. Muitas vezes com } \\
\text { uma abertura (arqueópilo). Através a } \\
\text { qual ocorre o encistamento. Jurássico- } \\
\text { Recente. } \\
\end{array}$ \\
\hline & & Prasinófitas & $\begin{array}{c}\text { Microfóssies produzidos } \\
\text { por pequenas algas } \\
\text { quadriflageladas (Divisão } \\
\text { Pyhophyta) } \\
\end{array}$ & $\begin{array}{l}\text { Maioria, como Tasmanites, são } \\
\text { esféricas; diâmetro } 50 \text { à } 2000 \text { ?m. } \\
\text { Pré-Cambriano-Recente. } \\
\end{array}$ \\
\hline & Zoomorfo & Palinoforaminífero & $\begin{array}{l}\text { Película interna que reveste } \\
\text { a carapaça de foraminíferos }\end{array}$ & $\begin{array}{l}\text { Parede interna quitinosa; marro; } \\
\text { câmaras menores muitas vezes mais } \\
\text { escuras. }\end{array}$ \\
\hline
\end{tabular}

matriz de correlação onde os valores mais próximos de +1 correspondem a correlações mais positivas e valores próximos de -1 a correlações menos positivas.

Foram realizados cálculos para a determinação dos parâmetros palinofaciológicos como a Razão fitoclasto opaco/ fitoclasto não-opaco e o Índice de Preservação de Fitoclastos (IPF). Ambos são utilizados para a obtenção de informações devido a diferenças nas condições de energia do ambiente, às variações proximal-distal, à história de transporte e aos processos de oxidação. Com o aumento das condições de mais alta energia a fração fitoclasto tende a tornar-se progressivamente dominada pelo material mais resistente (refratário). Isto é devido principalmente, a degradação mais acentuada sofrida pelos componentes mais frágeis (como por exemplo os fitoclastos não- 


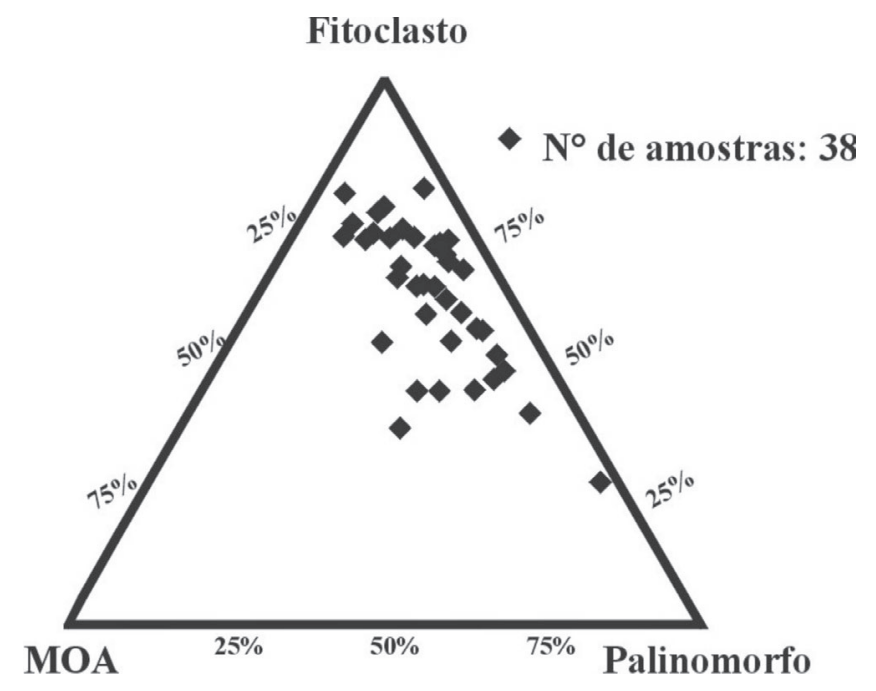

Figura 2. Diagrama ternário mostrando os percentuais de MOA, fitoclastos e palinomorfos das amostras analisadas.

Figure 2. Ternary AOM-phytoclast-palynomorphs diagram of the analysed samples.

opacos não-bioestruturados não-degradados, os fitoclastos nãoopacos listrados e as cutículas) e a conseqüente preservação seletiva dos tipos mais refratários (fitoclastos opacos e fitoclastos não-opacos bioestruturados do tipo traqueídico). Embora contínuo, este processo produziria mudanças claras na importância relativa de diferentes tipos de fitoclastos.

O IPF foi calculado utilizando a tendência de degradação de partículas lenhosas apresentada por Tyson (1995) pela determinação de uma escala numérica atribuída a cada tipo de fitoclasto (escore), o qual varia de 1 a 10. Esta escala nu- mérica é baseada na resistência à degradação dos componentes fitoclásticos, observada na análise por microscopia, com o número 1 representando o tipo de fitoclasto não-degradado menos refratário e o número 10 referenciando o material mais refratário.O IPF foi calculado de acordo com a seguinte expressão:

$\mathrm{IPF}=?\{[(\%$ tipo fitoclasto $/$ total de fitoclastos $) / 100] \mathrm{x}$ escore $\}$

\section{RESULTADOS}

\section{Análise visual e contagem dos componentes orgânicos particulados}

Na sucessão estudada observou-se o domínio do grupo fitoclastos no total de componentes da matéria orgânica (60,3\%), com os palinomorfos constituindo o segundo grupo em dominância $(27,6 \%)$ e subordinadamente MOA com valores percentuais médios inferiores a $15 \%$ (Figura 2).

O grupo dos fitoclastos constitui-se principalmente de cutículas (47,5\% do total de fitoclastos), com colorações de fluorescência variando do amarelo bem claro ao laranja escuro e partículas sem fluorescência. Segundo Habib (1982), através de leques submarinos quantidades significativas de cutículas atingem áreas profundas da bacia e são depositadas a partir dos cânios. Boulter \& Riddick (1986) observaram que as cutículas são relativamente mais abundantes em regiões de mais alta energia em leques submarinos, especialmente nos canais arenosos. Eles sugerem que este material flutuante passa normalmente pelos leques, e é rapidamente trapeado pela rápida sedimentação durante deposição de areias por fluxos gravitacionais.

Os fitoclastos não-opacos não-bioestruturados apresentaram média percentual de $15,5 \%$, os fitoclastos opacos variaram de

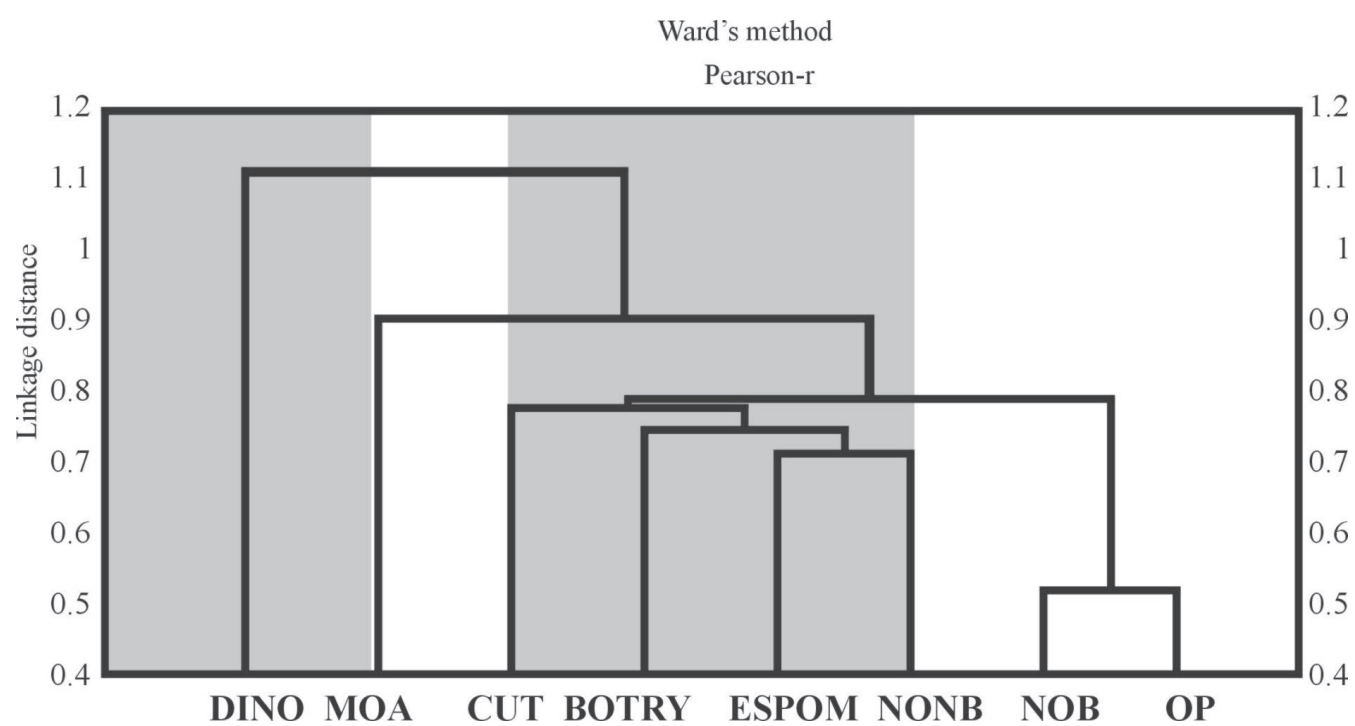

Figura 3. Dendograma produzido pela análise de agrupamento modo- $R$ para os grupos e subgrupos de componentes da matéria orgânica do testemunho de sondagem BU-91-GL-05.

Abreviaturas: BOTRY: Botryococcus; DINO: dinoflagelados; MOA: matéria orgânica amorfa; OP: fitoclastos opacos; NONB: fitoclastos não-opacos não- bioestruturados; CUT: cutículas; NOB: fitoclastos não-opacos bioestruturados; ESPOM: esporomorfos.

Figure 3. R-mode cluster analysis for the organic matter groups and sub-groups of the BU-91-GL-05 well.

Abreviattions: BOTRY: Botryococcus; DINO: dinoflagellates; AOM: Amorphous organic matter ; OP: Opaque phytoclast; NONB: Nonopaque non- biostructured phytoclast; CUT: Cuticles; NOB: Non-opaque biostructured phytoclast; SPOM: Sporomorphs . 
2,3 a 26,7\% e os demais fitoclastos não-opacos bioestruturados com média percentual de $26,7 \%$ do total de fitoclastos.

A fração de palinomorfos mostrou os esporomorfos (esporos e grão de pólen) como componentes dominantes (7,5 a 89,3\% do total de palinomorfos). Os esporomorfos apresentaram colorações de fluorescência do amarelo-esverdeado ao laranja claro, mas por vezes apresentaram-se degradados com coloração de fluorescência fraca. O subgrupo dos esporomorfos caracterizou-se por elevados percentuais de esporos $(7,1$ a 77,4\%) em relação aos grãos de pólen $(0,0$ $25,7 \%$ ). Os dinoflagelados dominaram a assembléia do microplâncton de parede orgânica marinho (média 28,06\%) com fluorescência amarela forte ao laranja médio.

O microplâncton de parede orgânica de água doce, algas do gênero Botryococcus, totalizou 10,35\% do total de palinomorfos e exibiu colônias e estruturas internas visíveis e identificáveis com fluorescência amarela intenso.

A MOA ocorreu como grupo minoritário, sendo constituída principalmente por MOA homogênea fluorescente (amarelo claro ao castanho escuro) com média percentual 41,6\% do total de MOA, seguida de resina (33,2\% de média), 22,7\% de MOA homogênea não fluorescente e subordinadamente MOA heterogênea com valores inferiores a $3 \%$.

Prasinófitas, Pediastrum, Pseudoschizea e palinoforaminíferos apareceram como ocorrências localizadas. Ainda foi registrada a ocorrência de ovos de copépodas.

\section{Análise de agrupamento modo-R e r-Peason}

O dendograma obtido pela análise de agrupamento modo$R$ revelou a separação do componente marinho (dinoflagelados - subgrupo I) dos demais subgrupos (matéria orgânica amorfa - subgrupo II e componentes continentais subgrupos III e IV) (Figura 3).

Através da matriz de correlação ( $r$-Pearson - TABELA 2) pode-se observar que as similaridades entre os componentes orgânicos estão relacionadas às tendências de deposição e ao equilíbrio hidrodinâmico das partículas. Os componentes orgânicos com tendências de distribuição em fácies mais proximais (Botryococcus, fitoclastos não-opacos nãobioestruturados, cutículas e esporomorfos) apresentaram correlações mais positivas, enquanto que as correlações tornamse mais negativas entre os componentes orgânicos com distribuição preferencialmente em fácies mais distais (fitoclastos opacos, fitoclastos não-opacos bioestruturados - tecidos lenhosos mais refratários e dinoflagelados). Notou-se que os fitoclastos opacos e fitoclastos não-opacos bioestruturados apresentaram correlações mais positivas entre si do que com os dinoflagelados. Este fato pode ser explicado, uma vez que esses componentes orgânicos mais refratários possuem a mesma origem e os mesmos processos de deposição seletiva.

\section{Análise de agrupamento modo- $Q$}

O dendograma produzido pela análise de agrupamento pelo modo $-Q$ revelou 5 agrupamentos. Esses agrupamentos, plotados estratigraficamente, possibilitaram a identificação de 5 intervalos (Figura 4). Os resultados dos valores percentuais da contagem 2 (contagem somente no grupo dos palinomorfos) também foram submetidos ao agrupamento modo- $Q$. Os intervalos obtidos pelas duas contagens coincidiram, mas observou-se um refinamento no intervalo 5, subdividindo-se este em mais 1 intervalo; totalizando 6 intervalos. Desta forma, a partir da subdivisão da seção sedimentar em intervalos foi possível observar características palinofaciológicas e organogeoquímicas distintas.

\section{Razões e parâmetros palinofaciológicos e organogeoquímicos}

Os valores da razão fitoclastos opacos/não-opacos (OP/

Tabela 2. Correlação de matrizes (coeficiente $r$-Pearson) para as amostras analisadas do testemunho de sondagem BU-91-GL-05 Abreviaturas: BOTRY: Botryococcus; DINO: dinoflagelados; MOA: matéria orgânica amorfa; OP: fitoclastos opacos; NONB: fitoclastos não-opacos não- bioestruturados; CUT: cutículas; NOB: fitoclastos não-opacos bioestruturados; ESPOM: esporomorfos. Table 2. Matrix correlation ( $r$-Pearson coeficient) for the studied samples of theBU-91-GL-05 well.

Abreviattions: BOTRY: Botryococcus; DINO: dinoflagellates; AOM: Amorphous organic matter ; OP: Opaque phytoclast; NONB: Nonopaque non- biostructured phytoclast; CUT: Cuticles; NOB: Non-opaque biostructured phytoclast; SPOM: Sporomorphs

\begin{tabular}{l|cccccccc}
\hline Variáveis & BOTRY & DINO & MOA & OP & NONB & CUT & NOB & ESPOM \\
\hline BOTRY & 1,00 & $-0,38$ & $-0,5$ & $-0,38$ & 0,7 & 0,23 & $-0,5$ & 0,26 \\
DINO & $-0,38$ & 1,00 & $-0,16$ & $-0,11$ & $-0,26$ & $-0,38$ & $-0,27$ & $-0,18$ \\
MOA & 0,5 & $-0,16$ & 1,00 & $-0,9$ & $-0,34$ & 0,10 & $-0,15$ & $-0,23$ \\
OP & $-0,38$ & $-0,11$ & $-0,9$ & 1,00 & 0,21 & $-0,43$ & 0,48 & $-0,29$ \\
NONB & 0,7 & $-0,26$ & $-0,34$ & 0,21 & 1,00 & $-0,13$ & $-0,20$ & 0,29 \\
CUT & 0,23 & $-0,38$ & 0,1 & $-0,43$ & $-0,13$ & 1,00 & $-0,03$ & $-0,32$ \\
NOB & $-0,5$ & $-0,27$ & $-0,15$ & 0,48 & $-0,20$ & $-0,03$ & 1,00 & $-0,54$ \\
ESPOM & 0,26 & $-0,18$ & $-0,23$ & $-0,29$ & 0,29 & $-0,32$ & $-0,54$ & 1,00 \\
\hline
\end{tabular}




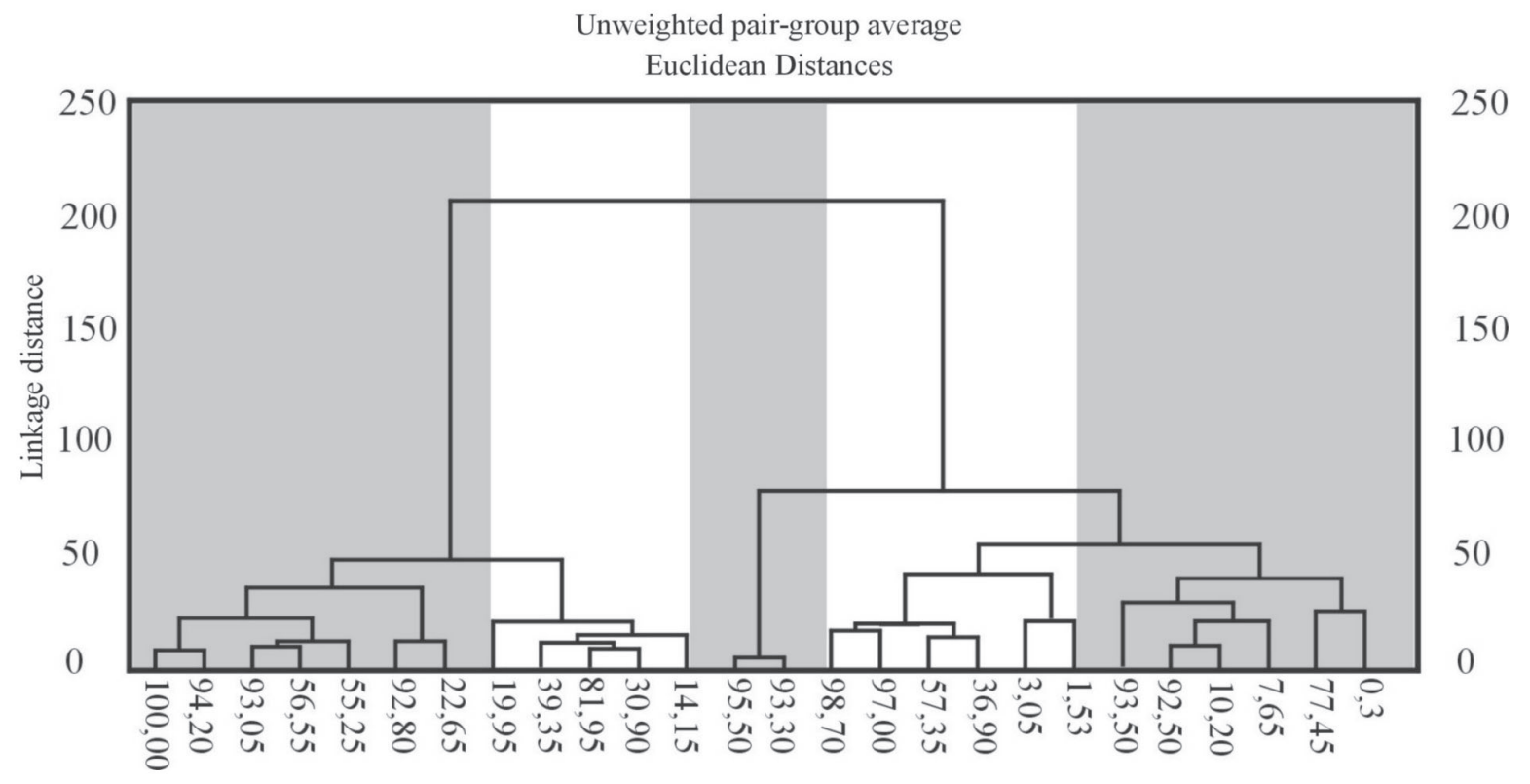

Figura 4. Dendograma produzido pela análise de agrupamento modo- $Q$ para os grupos e subgrupos de componentes da matéria orgânica do testemunho de sondagem BU-91-GL-05.

Figure 4. Q-mode cluster analysis for the organic matter groups and sub-groups of the BU-91-GL-05 well.

Tabela 3. Relações entre fácies orgânica e palinofácies para cada intervalo determinado pela análise de agrupamento modo- $Q$ para 0 conjunto de amostras analisadas do poço BU-91GL-05. *Taxa de sedimentação (Vicalvi, 1999): 0,95 a 1,10 m: $10 \mathrm{~cm} / 1000$ anos; 0,10 a 75 m: 146 e 158 cm/1000 anos; 75 m a 124 m: 113 cm/1000 anos; 124 a 150 m: ? COT- \% Carbono Orgânico Total; IH - índice de hidrogênio em mg Hc/ g Rocha; \% M.O.A. - \% de matéria orgânica amorfa do total de matéria orgânica ; \% Fit - \% de fitoclastos do total de matéria orgânica; MOA Fluor - matrix de fluorescência da mat'ria orgânica amorfa; OP/NO - razão opaco/não-opaco; Fácies Orgânica - Jones, 1987 e Tyson, 1995: BC- subdivisão da fácies anóxica-disóxica; C - fácies proximal flúvio-deltaica a pró-deltaíca; CD - fácies de plataforma óxica; D - fácies altamente óxica, distal e lentamente depositada.

Table 3. Relationship between organic facies and palynofacies for intervals defined by Q-mode cluster analysis for the studied samples of the BU-91-GL-05 well. *Sediment accumulation rate (Vicalvi, 1999): 0,95 a 1,10 m: 10cm/1000 years; 0,10 a $75 \mathrm{~m}: 146$ e $158 \mathrm{~cm} / 1000$ years; 75 m a 124 m: 113 cm/1000 years; 124 a 150 m: ? TOC- \% Total Organic Carbon; HI - hydrocarbon index (mg Hc/ g rock); \% AOM - \% amorphous organic matter of organic matter; \% Phy - \% phytoclast of organic matter; AOM fluor. - amorphous organic matter matrix fluorescence; OP/NO - opaque: non-opaque phytoclast; Organic Facies - Jones, 1987 and Tyson, 1995: BC- anoxic-dysoxic facies; C proximal fluvio-deltaic to prodeltaic facies; $C D$ - oxic shelf facies; D - highly oxic, distal and slowly deposited facies.

\begin{tabular}{l|cccccc}
\hline \multicolumn{1}{c}{ Palinof. } & Intervalo I & Intervalo II & Intervalo III & Intervalo IV & Intervalo Va & Intervalo Vb \\
\hline $\begin{array}{l}\text { \% COT } \\
\text { IH }\end{array}$ & $\mathrm{CD} / \mathrm{D}$ & $\mathrm{CD} / \mathrm{D}$ & $\mathrm{CD} / \mathrm{D}$ & $\mathrm{CD} / \mathrm{D}$ & $\mathrm{CD} / \mathrm{D}$ & $\mathrm{CD} / \mathrm{D}$ \\
$\%$ MOA & $?$ & $\mathrm{CD}$ & $\mathrm{CD}$ & $?$ & $\mathrm{CD}$ & $\mathrm{C}$ \\
MOA fluor. & $\mathrm{C} / \mathrm{CD} / \mathrm{D}$ & $\mathrm{BC}$ & $\mathrm{C} / \mathrm{CD} / \mathrm{D}$ & $\mathrm{C} / \mathrm{CD} / \mathrm{D}$ & $\mathrm{BC} / \mathrm{C}$ & $\mathrm{BC}$ \\
$\begin{array}{l}\text { \% Fit } \\
\text { OP/NO }\end{array}$ & $\mathrm{BC}$ & $\mathrm{C} / \mathrm{CD} / \mathrm{D}$ & $\mathrm{B} / \mathrm{BC}$ & $\mathrm{B} / \mathrm{BC}$ & $\mathrm{A} / \mathrm{AB}$ & $\mathrm{B} / \mathrm{BC}$ \\
$\begin{array}{l}\text { Regime de } \\
\text { oxigênio }\end{array}$ & $\mathrm{C} / \mathrm{CD} / \mathrm{D}$ & $\mathrm{C} / \mathrm{CD} / \mathrm{D}$ & $\mathrm{C} / \mathrm{CD} / \mathrm{D}$ & $\mathrm{C} / \mathrm{CD} / \mathrm{D}$ & $\mathrm{C} / \mathrm{CD} / \mathrm{D}$ & $\mathrm{C} / \mathrm{CD} / \mathrm{D}$ \\
$\begin{array}{l}\text { Taxa de } \\
\text { sedimentação* }\end{array}$ & $\mathrm{CD} / \mathrm{D}$ & $\mathrm{CD} / \mathrm{D}$ & $\mathrm{BC} / \mathrm{C}$ & $\mathrm{BC} / \mathrm{C}$ & $\mathrm{BC} / \mathrm{C}$ & $\mathrm{BC} / \mathrm{C}$ \\
$\begin{array}{l}\text { Palinof. } \\
\text { F. Org. }\end{array}$ & $?$ & $\mathrm{D}$ & $\mathrm{CD} / \mathrm{D}$ & $?$ & $\mathrm{CD} / \mathrm{D}$ & $\mathrm{C} / \mathrm{CD}$ \\
\hline
\end{tabular}


NO) variaram de 0,04 a 0,47 e os valores do Índice de Preservação dos Fitoclastos (IPF) de 0,8 a 5,0 com média de 2,81 e desvio padrão de 1,0 .

Os baixos valores encontrados nesses dois parâmetros refletem o predomínio de fitoclastos não-opacos (componentes menos refratários -cutículas), além de identificar uma deposição mais próxima da área fonte, uma vez que os fitoclastos opacos são formados por processos degradativos a partir dos fitoclastos não-opacos .

Os teores de COT variaram de 0,22 a $0,58 \%$. Esses baixos teores (inferiores a 2\%) são típicos de regiões de talude, ou seja, regiões com influência de correntes pobres em nutrientes e de correntes de fundo ricas em oxigênio (Henz et.al., 1996), além da presença de intensa atividade de organismos responsáveis por bioturbações nos sedimentos da sucessão sedimentar analisada.

Foram realizadas análises de pirólise Rock-Eval somente nas amostras que apresentaram teores de COT maior ou igual a $0,5 \%$ (total de 5 análises). Foram utilizados os resultados dos Índices de Hidrogênio (IH) e Oxigênio (IO), que

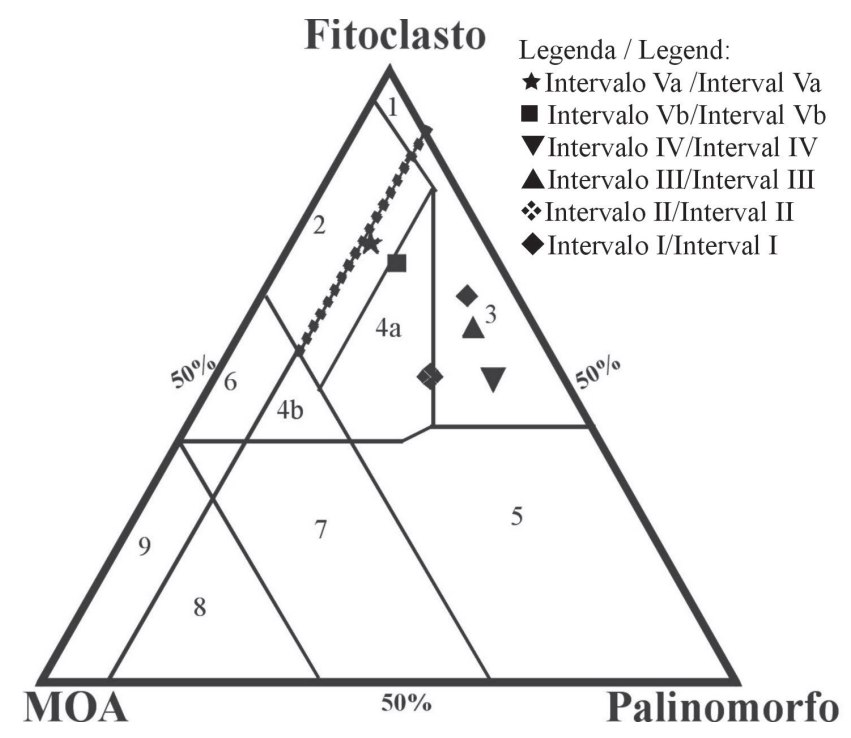

Figura 5. Diagrama ternário MOA-fitoclasto-palinormorfo para caracterização paleoambiental (Tyson, 1993) para o conjunto de amostras analisadas do poço BU-91-GL-05. 1: Bacia ou plataforma altamente proximal; 2: Bacia marginal disóxica-anóxica; 3: Plataforma óxica heterolítica ("plataforma proximal"); 4: Transição plataforma -bacia - 4a: dysóxica, 4b: subóxica-anóxica; 5: Plataforma óxica dominada por lama ("plataforma distal") ; 6: Plataforma proximal subóxica-anóxica; 7: Plataforma distal disóxica-anóxica; 8: Plataforma distal disóxica-anóxica; 9: Bacia distal subóxicaanóxica

Figure 5. Ternary AOM-phytoclast-palynomorph organic matter plot (based on Tyson, 1993). 1: Highly proximal shelf or basin; 2: Marginal dysoxic-anoxic basin; 3: Heterolithic oxic shelf ("proximal shelf"); 4: Shelf to basin transition - 4a: dysoxic, 4b: suboxicanoxic; 5: Mud-dominated oxic shelf ("distal shelf"); 6: Proximal suboxic-anoxic shelf; 7: Distal dysoxic-anoxic shelf; 8: Distal dysoxic-oxic shelf; 9: Distal suboxic-anoxic basin revelaram baixos valores de $\mathrm{IH}(<138 \mathrm{mgHC} / \mathrm{gCOT})$ e valores de $\mathrm{IO}$ entre 370,7 a 678,4 $\mathrm{mgCO}_{2} / \mathrm{gCOT}$, caracterizando o baixo grau de preservação da matéria orgânica devido, provavelmente, a grande disponibilidade de oxigênio no ambiente deposicional.

\section{Fácies orgânica}

A fácies orgânica, sensu Jones (1987), foi determinada segundo os parâmetros descritos por Tyson (1995) e Mendonça Filho (1999). Com base nesses parâmetros foi determinada, para seção analisada, uma variação organofaciológica entre as fácies BC (fácies disóxica-anóxica com um elevado conteúdo de fitoclastos), C (fácies proximal flúviodeltaica a pró-deltaica) e CD (fácies de plataforma óxica) (Tabela 3).

\section{Interpretação paleoambiental}

Considerando os principais parâmetros dos 3 grupos principais da assembléia de componentes da matéria orgânica, a maioria das características dos paleoambientes determinados são geralmente similares, sendo dominados por fitoclastos, porém com contribuições significativas de palinomorfos e com moderada taxa de preservação de MOA.

De acordo com o diagrama ternário representado na Figura 5, o qual utiliza os valores percentuais dos 3 grupos principais da matéria orgânica, os intervalos I, II,III e IV recaíram no campo 3, que apresenta uma assembléia de componentes da matéria orgânica correspondente àquela de-

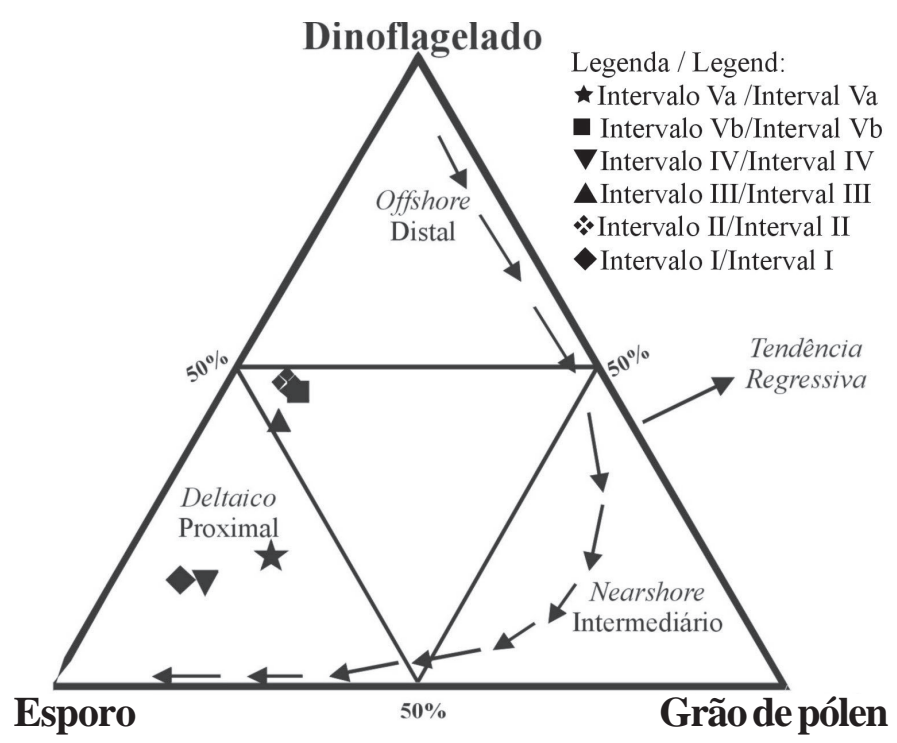

Figura 6. Diagrama ternário com os campos de tendências transgressivas-regressivas definidos pelos valores percentuais dos dinoflagelados, esporos e grãos de pólen para o poço BU-91GL-05 (Tyson, 1995).

Figure 6. Ternary dinoflagellate-spore-pollen palynomorph plot. Smoothed theoretical transgressive-regressive trens is indicated (based on Tyson, 1995). 
positada em plataforma óxica. O intervalo Va recaiu na interface dos campos 4 a e 2, sendo caracterizado por um paleoambiente de talude com regime disóxico. O intervalo Vb recaiu no campo 2, caracterizado por um conteúdo orgânico correspondente aquele de depositado em um paleoambiente de bacia marginal com condições disóxicaanóxica.

A figura 6 mostra os indicadores de tendências transgressiva-regressiva / proximal-distal baseado em Tyson (1995). Os valores médios percentuais de dinoflagelados, esporos e grãos de pólen sugerem que nesta sucessão os intervalos I, $\mathrm{IV}$ e $\mathrm{Vb}$ apresentaram tendências mais proximais em relação aos outros intervalos que mostraram tendências intermediárias (proximal deltaica-offshore).

\section{DISCUSSÃO}

Notou-se que as variações dos parâmetros palinofaciológicos e organogeoquímicos estão diretamente relacionadas aos intervalos de maior e menor influência de aporte de material orgânico de origem continental. Esta ciclicidade está provavelmente relacionada às oscilações do nível relativo do mar, reflexo de flutuações climáticas ocorridas nos últimos 150.000 anos AP, as quais influenciaram o suprimento de material orgânico, uma vez que interferiram principalmente na paleoceanografia da região analisada.
Estes resultados foram correlacionados com as subdivisões biocronológicas (foraminíferos) do Quaternário no Talude Continental na Bacia de Campos de Vicalvi (1999), que utilizou o mesmo testemunho de sondagem, aqui analisado, onde foram determinados dois períodos glaciais (zonas $\mathrm{Y}$ e $\mathrm{W}$ ) e dois interglaciais (zonas $\mathrm{Z}$ e X).

Nos períodos glaciais, com o conseqüente rebaixamento do nível relativo do mar, ocorrem condições mais proximais de deposição dos componentes orgânicos, o que pode estar relacionado com a exposição da plataforma e consequiente deposição de vários sistemas sedimentares que trazem um maior suprimento de material orgânico continental. Nos períodos interglaciais com o aumento do nível relativo do mar / temperatura, ocorre uma diminuição no suprimento de material orgânico de origem continental, uma vez que o aumento da coluna d'água / temperatura auxilia tanto no desenvolvimento da bioprodutividade do fitoplâncton marinho, como no processo de produção de material orgânico amorfo (atividade microbiológica)

Com a integração dos dados das análises de palinofácies e fácies orgânica foi possível traçar a curva de variação do nível relativo do mar para ao longo da seção sedimentar estudada, caracterizar as variações palinofaciológicas e organogeoquímicas para cada intervalo determinado pela análise de agrupamento modo- $Q$ e correlacionar com biozonas (foraminíferos) pré-estabelecidas (Figura 7):

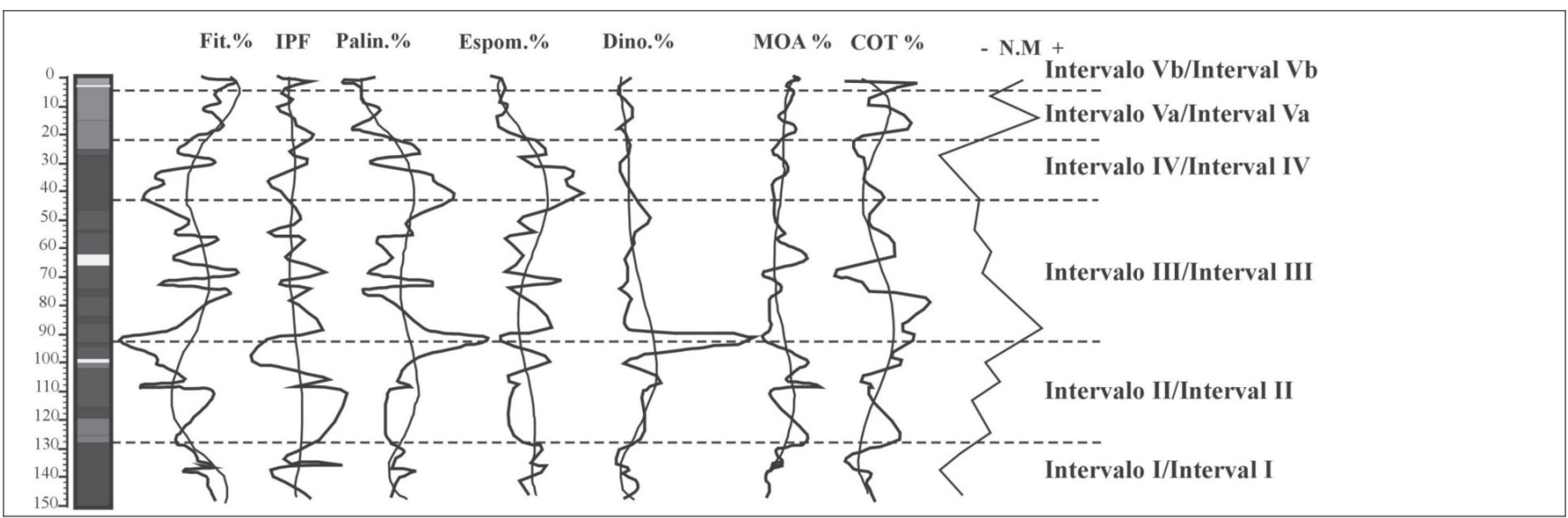

Legenda/Legend:

Areia lamosa bioturbada/Bioturbated muddy sand Areia siliciclástica/Siliciclastic sand

Lama oliva com intercalações arenosas/Olive mud/sand intercalations
Lama cinza esverdeada/Greenish-grey mud

Lama cinza escuro esverdeada/Dark greenish-grey mud

Lama cinza escuro esverdeada com intercalações arenosas/Dark greenish-grey mud/sand intercalations

Lama preta esverdeada /Greenish-black mud

Depósitos de escorregamento/Slumps deposits

(2) Linha de tendência/Trend line
Fit. $=$ Fitoclasto $/$ Phy $=$ Phytoclast

$\mathrm{PF}=$ İndice de Preservação de Fitoclasto $/$ PPI = Phytoclast Preservation Index

Palin. $=$ Palinomorfo $/$ Palin $=$ Palynomorph

Epom $=$ Esporomorfo $/$ Spom $=$ Sporomorph

Dino $=$ Dinoflagelado $/$ Dinoflagellate

MOA = Matéria Orgânica Amorfa / AOM = Amorphous Organic Matter

COT $=$ Carbono Orgânico Total $/$ TOC $=$ Total Organic Carbon $\mathrm{NM}=$ Nivel do Mar $/ \mathrm{SL}=$ Sea Level

Figura 7: Variações dos parâmetros palinofaciológicos e organogeoquímicos ao utilizadas para traçar a curva do nível relativo do mar para a seção sedimentar analisada e correlação com as biozonas determinadas por Vicalvi (1999). Intervalo I: Proximal - fácies de plataforma óxica; Intervalo II: Proximal - fácies de plataforma óxica; Intervalo III: Proximal/intermediário - fácies de plataforma óxica; Intervalo IV: Intermediário/distal - fácies de plataforma óxica-disóxica; Intervalo Va: Intermediário/distal - fácies de talude disóxica; Intervalo Vb: Intermediário/proximal - fácies bacia marginal disóxica

Figure 7: Palynofacies and organic geochemical parameters showing the sea level change and a correlation with the biozones from Vicalvi (1999). Interval I: Proximal - oxic shelf facies; Interval II: Proximal - oxic shelf facies; Interval III: Proximal/intermediary - Proximal - oxic shelf facies; Interval IV: Intermediary /distal - dysoxic-oxic shelf facies; Interval Va: Intermediary /distal dysoxic basin slope facies; Interval $\mathrm{Vb}$ : Intermediary /proximal marginal dysoxic basin facies 
Intervalo I (140.000-127.000 anos AP/Biozona W). Apresenta uma assembléia de componentes da matéria orgânica correspondente aquela depositada em paleoambiente de fácies de plataforma óxica com tendências proximais (flúviodeltaica), com tendência geral do nível relativo do mar de elevação. Apresenta predomínio de matéria orgânica continental e baixos percentuais de MOA, COT e IPF.

Intervalo II (127.000-84.000 anos AP/Biozona X). Apresenta uma assembléia de componentes da matéria orgânica que corresponde àquela depositada em paleoambiente de fácies de plataforma óxica com tendências proximais (flúvio- deltaicas). Tendência de elevação do nível relativo do mar. Ocorre aumento na média percentual de dinoflagelados, MOA, COT e IPF e diminuição dos valores percentuais de fitoclastos e esporomorfos.

Intervalos III e IV (84.000-42/45.000 anos AP/Biozona Y2/ início da Biozona Y1). Tendência de rebaixamento do nível relativo do mar. Apresenta deposição de matéria orgânica correspondente àquela encontrada em plataforma óxica com tendências intermediárias. Esses intervalos são marcados pelo aumento percentual dos fitoclastos, média percentual de esporomorfos bem maior que a média percentual de dinofla-

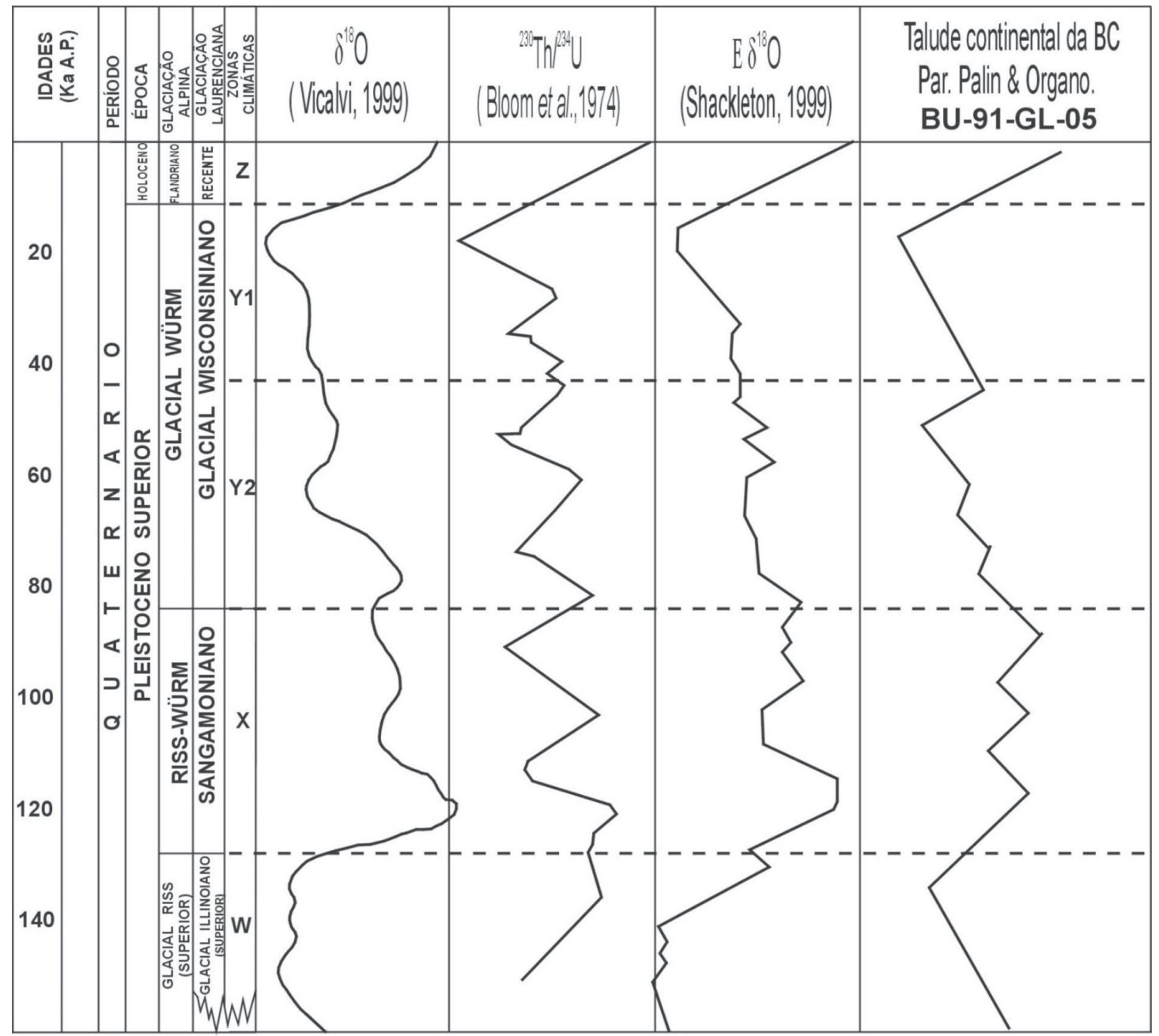

Curva de variação do nível do mar / Sea level trend

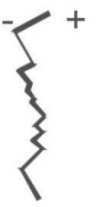

Figura 8. Correlação gráfica das tendências de variação eustática do nível do mar no Quaternário entre as curvas fornecidas por diferentes autores e resultados obtidos com os dados palinofaciológicos e organogeoquímicos no talude continental superior da Bacia de Campos.

Figure 8. Graphic correlation of the Quaternary sea level changes trends using palynofacies and organic parameters for the upper continental slope from Campos Basin. 
gelados e baixos percentuais de MOA, COT e IPF. Ocorrem, no intervalo III, picos de dinoflagelados e MOA que podem estar correlacionados a variações climáticas (temperaturas mais altas dentro de um episódio glacial), que auxiliaram na bioprodutividade do microplâncton marinho e na produção de MOA ou confinamento do ambiente ocasionando mudanças no regime de oxigênio / condições menos óxicas.

Intervalo Va (42/45.000-11.000 anos AP/ final da Biozona Y1). Corresponde ao paleoambiente de fácies de talude com regime disóxico com tendência intermediária. A tendência geral do nível relativo do mar é de elevação, porém com picos de rebaixamento. Ainda é marcado por valores percentuais elevados de fitoclastos, média percentual de esporomorfos bem maior que a média percentual de dinoflagelados. Devido as condições menos óxicas os valores percentuais de MOA são mais elevados .

Intervalo Vb (11.000 anos - recente/final da Biozona Y1/ Biozona Z). Neste intervalo a tendência do nível relativo do mar é de elevação. Como no intervalo anterior, ainda é marcado por valores percentuais elevados de fitoclastos, média percentual de esporomorfos bem maior que a média percentual de dinoflagelados. Apresenta uma assembléia de componentes da matéria orgânica que corresponde àquela depositada em paleoambiente de fácies de bacia marginal com regime disóxico com tendências intermediárias.

A passagem Plesitoceno/Holoceno é marcada pela subida do nível relativo do mar. Esta passagem é caracterizada pela mudança no suprimento de matéria orgânica: queda na média percentual de fitoclastos, presença de um número maior de componentes marinhos (aumento percentual de dinoflagelados de aproximadamente $0,0 \%$ para $15 \%$ ), aumento de MOA, COT e IPF.

A curva de variação eustática do nível do mar no Quaternário, confeccionada a partir das variações dos parâmetros palinofaciológicos e organogeoquímicos, para a seção sedimentar analisada, foi correlacionada com as curvas propostas por Vicalvi (1999), o qual utiliza dados de $\mathrm{d}^{18} \mathrm{O}$, com dados de ${ }^{230} \mathrm{Th} /{ }^{234} \mathrm{U}$ (Bloom et al, 1974) e, com os dados de $\mathrm{d}^{18} \mathrm{O}$ Shackleton (1987). Tal correlação mostrou-se muito positiva, apresentando elevado grau de correspondência (Figura 8).

\section{CONCLUSÕES}

As variações no suprimento de material orgânico estão relacionadas às oscilações do nível relativo do mar, reflexo de flutuações climáticas, ocorridas nos últimos 150.000 anos AP. Esses fatores climáticos ocasionaram mudanças no aporte de partículas orgânicas e variações no regime de oxigênio da área de estudo; influenciando desta forma, os fatores gerais que produziram, acumularam e preservaram os grupos e subgrupos de componentes da matéria orgânica.

Os resultados obtidos através da aplicação de Palinofácies e Fácies Orgânica, integrando diferentes metodologias na análise de sedimentos quaternários do talude continental superior da Bacia de Campos, mostraram que a aplicação desse estudo constitui uma valiosa e eficaz ferramenta na reconstrução de paleoambientes e na determinação das condições deposicionais da matéria orgânica sedimentar.

\section{AGRADECIMENTOS}

Ao CNPq/CTPETRO (Projeto n $\left.{ }^{\circ} 460-492 / 000-04\right)$ pelo apoio financeiro; ao Laboratório de Geologia/Unidade de Negócio de Exploração e Produção da Bacia de Campos/ PETROBRAS pela liberação das amostras; à Gerência de BioEstratigrafia e Paleoecologia (CENPES/PETROBRAS) pelo apoio logístico e financeiro; e à Gerência de Geoquímica / CENPES/PETROBRAS pelo apoio analítico.

\section{REFERÊNCIAS}

Assis, JR. F. 2000. New Organic Matter Isolation Procedure and Equipment. In: LATIN AMERICAN CONGRESS ON ORGANIC GEOCHEMISTRY, 7, 2000. Anais, Foz do Iguaçu, ALAGO, p. 355-357.

Boulter, M.C. \& Riddick, A. 1986. Classification and analysis of palinodebris from the Palaeocene sediments of the Forties Field. Sedimentology, 33: 871-86.

Caddah, L.F.G; Kowsmann, R.O.\& Viana, A.R. 1998. Slope sedimentary facies associated with Pleistocene and Holocene sea-level changes, Campos Basin, southeast Brazilian Margin. Sedimentary Geology, 115: 159-174.

Habib, D. 1982. Sedimentary supply origin of cretaceous black shales: In: S.O Schlanger, \& M.B. Cita, (eds.) Nature and origin of Cretaceous Carbon-rich Facies, Academic Press, p. 113-27.

Henz, G.I.; Kowsmann, R.O \& Meniconi, M.F.G. 1996. A petrografia orgânica de amostras do quaternário da Bacia de Campos. In: CONGRESSO BRASILEIRO DE GEOLOGIA, 38, 1996. Anais, Camburiú, SBG, v.1, p. 416-417.

Jones, R.W. 1987. Organic Facies: In: J. BROOKS, \& D. WELTE, (eds.) Advances in Petroleum Geochemistry 2, Academic Press, p. 1-90.

Mendonça Filho, J.G. 1999. Aplicação de estudos de palinofácies e fácies orgânica em rochas do Paleozóico da Bacia do Paraná, Sul do Brasil. Programa de Pós-Graduação em Geociências, Universidade Federal do Rio Grande do Sul, Tese de Doutorado, 338 p.

Mendonça Filho, J.G; Carvalho, M.A. \& Menezes, T.R., 2002. Palinofácies. In: Técnicas e procedimentos de trabalho com fósseis e formas modernas comparativas, Editora Unisinos, $\mathrm{p}$. 20-24.

Tyson, R.V. 1993. Palynofacies Analysis. In: D.J. Jenkins (ed.) Applied Micropaleontology, Kluwer Academic Publishers, p. 153-191.

Tyson, R.V. 1995. Sedimentary Organic Matter: Organic facies and palynofacies. $1^{\mathrm{a}}$ ed. Londres, Chapman \& Hall, $615 \mathrm{p}$.

Tuweni, A.O. \& Tyson, R.V. 1994. Organic facies variations in the Westbury Formation (Rhaetic Bristol Channel, SW England). Organic Geochemical, 21(10/11): 1001-1014.

Viana, A.R. \& Faugères, J.C. 1998. Upper slope sand deposits: the example of Campos Basin, a latest Pleistocene-Holocene record of the interaction between alongslope and downslope currents. In: M.S. Stoeker, D. Evans, \& A. Cramp (eds.) Geologycal Processes on Continental Margins: Sedimentation, Mass-Wasting and Stability, Geological Society, Special Publications, 129: 287-316. 
Viana, A.R.; Faugères, J.C.; Kowsmann,R.O; Lima, J.A.M.; Caddah, L.F.G. \& Rizzo J.G. 1998. Hydrology, morphology and sedimentology of the Campos continental margin, offshore Brazil. Sedimentary Geology, 115: 133-157.
Vicalvi, M.A.1999. Zoneamento Bioestratigráfico e Paleoclimático do Quaternário Superior do Talude da Bacia de Campos e Platô de São Paulo adjacente, com base em Foraminíferos Planctônicos. Programa de Pós-Graduação em Geologia, Universidade Federal do Rio de Janeiro, Tese de Doutorado, 184 p. 